\title{
Characterization of Interstitial Stem Cells in Hydra by Cloning
}

\author{
Charles N. David and Susan Murphy \\ Department of Molecular Biology, Albert Einstein College of Medicine, Bronx, New York 10461 \\ Received December 29, 1976; accepted in revised form March 18, 1977
}

\begin{abstract}
A procedure has been developed for cloning interstitial stem cells from hydra. Clones are prepared by introducing small numbers of viable cells into aggregates of nitrogen mustardinactivated host tissue. Clones derived from added stem cells are identified after 1-2 weeks of growth by staining with toluidine blue. The incidence of clones increases with increasing input of viable cells according to one-hit Poisson statistics, indicating that clones arise from single cells. After correction for cell losses in the procedure, about $1.2 \%$ of the input cells are found to form clones. This compares with estimates from in vivo experiments of about $4 \%$ stem cells in whole hydra [David, C. N., and Gierer, A. (1974). Cell cycle kinetics and development of Hydra attenuata. III. Nerve and nematocyte differentiation. J. Cell Sci. 16, 359-375.]

Differentiation of nematocytes and nerve cells in clones was analyzed by labeling precursors with $\left[{ }^{3} \mathrm{H}\right]$ thymidine and scoring labeled nerves and nematocytes 2 days later. Nine clones examined in this way contained both differentiated nerve cells and nematocytes, demonstrating that the interstitial stem cell is multipotent. This result suggests that the observed localization of nerve and nematocyte differentiation in whole hydra probably occurs at the level of stemcell determination. The observation that differentiated cells occur very early in clone development suggests that a stem cell's decision to proliferate or differentiate is regulated by shortrange feedback signals which are already saturated in young clones.
\end{abstract}

\section{INTRODUCTION}

The differentiation of cells in patterns is a major event in embryogenesis. Little is known about the mechanisms of this process beyond the broad statement that cells seem to "know" their position in a larger structure and use that information to choose specific pathways of differentiation from a larger repertoire of possible pathways (Wolpert, 1971). The simple metazoan hydra provides a model system in which to investigate this regionalization of cell differentiation, since formation of nematocytes is restricted to the central region of the body column, while nerve differentiation occurs primarily at the ends of the body column near hypostome and basal disk (David and Challoner, 1974; David and Gierer, 1974). The purpose of the present investigation is to examine the properties of the nerve and nematocyte precursor, the interstitial cell, with the ultimate objective of understanding the control mechanisms involved in its differentiation.

Nerve and nematocyte differentiation occur continuously in asexually growing hydra and have been extensively characterized by microscopy (Slautterback and Fawcett, 1959; Lentz, 1966; Davis, 1969, 1971) and cell-flow analysis (David and Gierer, 1974). A continuous supply of precursors for differentiation is maintained by a population of interstitial "stem" cells, $60 \%$ of whose daughters cells remain stem cells, while $40 \%$ initiate either nerve or nematocyte differentiation. To sustain the measured production rate of about 500 nerves and 1800 nematocyte clusters per day per hydra, about 3600 stem cells are required or about $4 \%$ of the total cells in a hydra. David and Gierer (1974) concluded that stem cells are a subpopulation of a particular class of large interstitial cells which occur singly and in pairs.

It has not yet been established whether 
the stem cells in hydra consist of a single population of multipotent cells or whether there are several morphologically identical but functionally distinct subpopulations, e.g., stem cells for nerve differentiation, stem cells for nematocyte differentiation, and stem cells for the germ line. An answer to this question is essential to understanding the patterns of nerve and nematocyte differentiation in hydra. If stem cells are truly multipotent, the spatial patterns of nerve and nematocyte formation observed in the body column would be due to positional signals controlling stem-cell determination. If distinct nerve and nematocyte stem cells exist, these two determined populations must somehow be localized at different sites in the body column.

The differentiation potential of individual stem cells can be directly assayed if these cells are cloned. Since hydra cells cannot yet be grown in vitro (Trenkner et al., 1973), we have developed an in vivo cloning procedure which is analogous to Till and McCulloch's (1961) method for culturing bone marrow stem cells in myeloid tissues of lethally $X$-irradiated mice. In our procedure, hydra are treated with nitrogen mustard (NM) which eliminates interstitial cells from their tissues (Diehl and Burnett, 1964). We use such animals as hosts in which to culture live interstitial cells, employing a disaggregationreaggregation technique (Gierer et al., 1972) to introduce the live cells into the nitrogen mustard-treated tissue.

Here we describe the growth of clones from single interstitial cells in this system. We have determined the number of clone-forming cells (stem cells) in normal hydra tissue and have observed both nerve and nematocyte differentiation in clones derived from single stem cells. We have further observed that differentiation is initiated very early in clone growth and suggest that feedback signals regulating the proportion of differentiating versus proliferating stem-cell daughters are probably of short range. Finally, we suggest that the in vivo culturing procedure has a number of further applications in assaying differentiating cells and cloning other hydra cell types.

\section{MATERIALS AND METHODS}

Hydra attenuata were used for all experiments. Feeding and culture conditions have been described (David and Gierer, 1974).

Nitrogen mustard (NM) treatment. Hydra were treated for $10 \mathrm{~min}$ at $20-21^{\circ} \mathrm{C}$ with $0.01 \%$ freshly prepared nitrogen mustard (2,2 dichloro- $N$-methyldiethylamine $\mathrm{HCl}$; Sigma Chemical Co.) (Diehl and Burnett, 1964). To stop the treatment, hydra were washed four times with hydra water. Nitrogen mustard treatment causes progressive elimination of interstitial cells from tissue during the 6-8 days following treatment. Feeding accelerates the elimination of interstitial cells. Most cloning experiments were started 1 day after NM treatment and were stained for clones 10 12 days later, after host interstitial cells were eliminated. In experiments requiring host tissue without interstitial cells at the start of cloning, hydra were pretreated with NM, fed 1 day later, and used for cloning experiments 6-7 days thereafter.

Cloning procedure: Preparation of dissociated cells and reaggregation. Suspensions of dissociated cells were prepared by shearing large numbers of hydra in an isotonic cell culture medium as previously described (Gierer et al., 1972). Dissociation of small amounts of hydra tissue (pieces of hydra or a few wholc animals) was achieved by adding nitrogen mustardtreated animals as carrier and dissociating the mixture. Under these conditions, the recovery of clone-forming cells from the live tissue was quantitative.

Interstitial cells occur in suspensions of dissociated cells almost completely as single cells. In a representative preparation, $95 \%$ of interstitial cells were single, $5 \%$ occurred in pairs, and $<0.5 \%$ in groups of 
3-5. The pairs and small clusters appear microscopically to be the result of incomplete dissociation of interstitial cell clusters occurring in vivo.

To prepare interstitial cell clones, small numbers of dissociated live cells were mixed with $2 \times 10^{5}$ dissociated nitrogen mustard-treated cells, and the mixture was pelleted. Regeneration of the pellets proceeded as described previously for normal cell aggregates (Gierer et al., 1972).

Interstitial cell clones were identified by staining alcohol-fixed aggregates with $0.025 \%$ toluidine blue (Diehl and Burnett, 1964) and examining whole amounts at $160 \times$ or $400 \times$. Interstitial cells stain darkly against weakly staining host tissue. By changing focus it is possible to score clones throughout an aggregate.

Assay of clone-forming cells (stem cells). Two procedures were used to quantitate clone-forming cells in samples. (1) $P(0)$ procedure: 20-30 aggregates were prepared having $0.5-1$ clone/aggregate. The average number of clone-forming cells per sample was calculated from the Poisson distribution using the measured fraction of aggregates containing zero clones, $P(0)$. This method does not depend on discriminating single from double clones in aggregates, and thus, can be used late in clone development (10-15 days) when clones are large. (2) Total clone procedure: The total number of clones was counted in 20-30 aggregates. Young (5-6 day) clones were scored to avoid counting two clones as one. In such experiments, hosts pretreated with nitrogen mustard were used so as not to

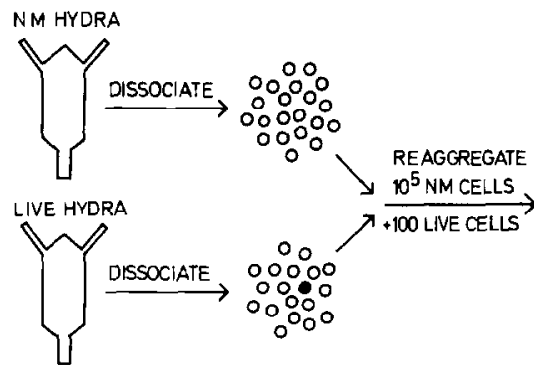

confuse clones with dying interstitial cells of the host.

The two procedures yielded results comparable to within $30 \%$. The variability between replicate assays and equivalent assays performed on different days was also about $\pm 30 \%$.

Cell counting. Suspensions of dissociated cells were counted in a Neubauer hemocytometer, or, more frequently, parallel samples of hydra tissue were dissociated and counted by the maceration procedure (David, 1973) which permits cell counting as well as identification of all cell types. In maceration preparations, interstitial cells occur as large single cells or pairs of cells and as smaller cells in clusters of 4,8 , and 16 cells. The large cells are referred to as $1 \mathrm{~s}$ and $2 \mathrm{~s}$ and contain the class of cells forming clones. The latter procedure was followed in all experiments in which clones per input 1s and 2s were determined.

Isotopic labeling and autoradiography. Whole hydra and aggregates were labeled by injecting small volumes $(0.1 \mu \mathrm{l})$ of $\left[{ }^{3} \mathrm{H}\right]$ thymidine $(100 \mu \mathrm{Ci} / \mathrm{ml} ; 30 \mathrm{Ci} / \mathrm{mmole})$ into the gastric cavity. To analyze labeled cells, labeled tissue was dissociated by maceration, dried on microscope slides, and covered with Kodak AR10 stripping film for autoradiography (David and Campbell, 1972).

\section{RESULTS}

\section{Preparation of Stem Cell Clones}

The procedure for cloning stem cells is outlined in Fig. 1. Suspensions of normal and nitrogen mustard (NM)-treated cells

FIG. 1. Schematic representation of the procedure for cloning interstitial stem cells (O) in nitrogen mustard (NM)-treated host aggregates. 
are prepared, and aliquots containing 50 400 normal cells are added to aliquots containing about $2 \times 10^{5} \mathrm{NM}$ cells. The mixed cell suspensions are then centrifuged, and the pellets are transferred to petri dishes containing fresh medium. The aggregates regenerate during the next several days, during which time interstitial cell clones develop. Figure 2 shows early and late stages of clone development in preparations stained with toluidine blue. Control aggregates to which no live cells are added do not contain interstitial cell clones.

\section{Experiments on the Origin of Clones}

The simplest explanation for the origin of clones is that they are derived from interstitial stem cells in the added live-cell suspension. The absence of clones in control aggregates to which no live cells are added supports this conclusion. However, two alternative explanations must be considered: (1) that clones arise by "reactivation" of host cells in the presence of live cells; and (2) that clones are derived from some cell other than stem cells in the livecell suspensions, e.g., epithelial or gland cells. To demonstrate directly that the clones are derived from the added live cells and not host cells, we have labeled the input cells with $\left[{ }^{3} \mathrm{H}\right]$ thymidine and have scored labeled cells in clones by autoradiography. Table 1 shows that $46 \%$ of the input stem cell population was labeled and that $48 \%$ of the cells in 3-day clones were also labeled. This eliminates host cells, which were unlabeled, as the source of clones.

The results in Table 1 suggest, furthermore, that clones are derived from interstitial cells in the live-cell suspension, since the clones have the same specific labeling as the input interstitial-cell population, namely, $48 \%$, as compared to 12.5 and $6.5 \%$ labeling, respectively, in the input epithelial and gland-cell populations. To obtain decisive evidence on this point, we have developed a line of proliferating stem cells (and their differentiated derivatives) free of proliferating epithelial and gland cells. The stem-cell line is maintained in NM host aggregates by serial transfer every 2 weeks (David and Murphy, in preparation). When such aggre-
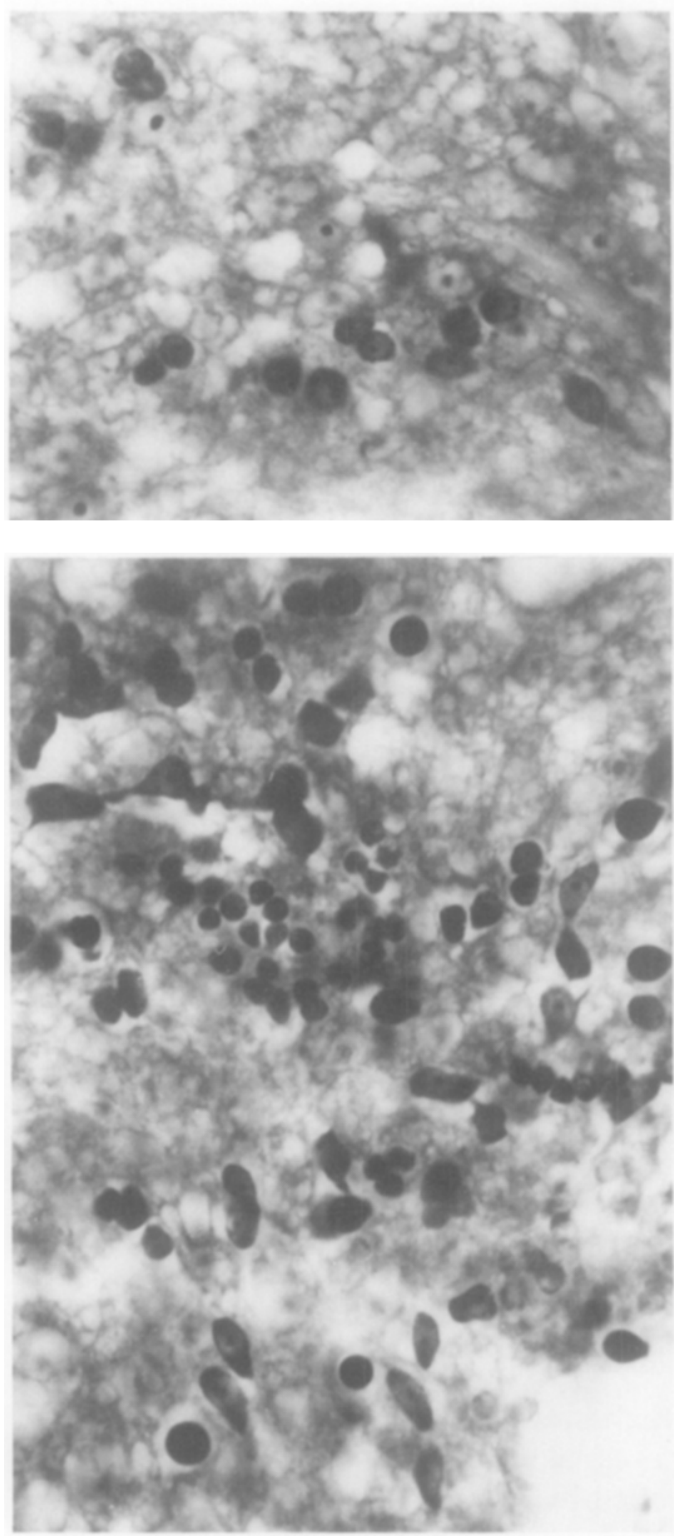

FIg. 2. Toluidine blue-stained aggregates showing two stages in the growth of stem-cell clones. Darkly stained interstitial cells stand out against weakly stained host tissue. Top: 4-day clone containing 16 cells, primarily large interstitial cells in pairs. Bottom: 10-day clone containing about 150 cells including interstitial cell clusters and differentiating nematoblasts. Host interstitial cells were eliminated by pretreatment with nitrogen mustard. $\times 300$. 
TABLE 1

Recovery of Labeled INpUt Cells in 3-Day INTerstitial Cell Clones ${ }^{a}$

\begin{tabular}{lccc}
\hline & \multicolumn{3}{c}{ Labeling index } \\
\cline { 2 - 4 } & $\begin{array}{c}\text { Large } \\
\text { inter- } \\
\text { stitial } \\
\text { cells } \\
(1 \mathrm{~s}+2 \mathrm{~s})\end{array}$ & $\begin{array}{c}\text { Epithelial } \\
\text { cells }\end{array}$ & $\begin{array}{c}\text { Gland } \\
\text { cells }\end{array}$ \\
\hline $\begin{array}{l}\text { Input cells }\left(t_{0}\right) \\
\begin{array}{l}\text { Interstitial cell } \\
\text { clones }\left(t_{3}\right)\end{array}\end{array}$ & $46 \%$ & $12.5 \%$ & $6.5 \%$ \\
\hline
\end{tabular}

${ }^{a}$ Twenty hydra were pulse-labeled for $2 \mathrm{hr}$ with $\left[2{ }^{14} \mathrm{C}\right]$ thymidine. Four hydra were macerated immediately after labeling $\left(t_{0}\right)$ to determine the labeling indices of the interstitial cell $(1 s+2 s)$, epithelial cells, and gland cells of the input cells. The remaining 16 hydra were dissociated with carrier NM hydra and were added to NM aggregates at a concentration of 20 clone-forming cells/aggregate. On Day 3 , aggregates were macerated, and the labeling index of interstitial cells ( $1 s+2 s$ ) was determined by autoradiography. Dilution of label due to cell division was compensated by longer exposure times. Pretreated NM host hydra was used throughout the experiment. The labeling index of epithelial and gland cells in aggregates was not determined (nd) because of the presence of host cells.

gates containing the stem-cell line are dissociated and added at low input to fresh NM host aggregates, the cloning efficiency of added interstitial cells is unchanged relative to dissociated normal hydra (Table 2). This proves that stem-cell clones are derived from interstitial cells in the heterogeneous mixture of cell types present in the input live-cell suspensions.

\section{Statistics of Clone Formation}

As the number of input live cells per aggregate increases, the fraction of aggregates containing clones increases asymptotically to $100 \%$. If a single cell is sufficient to found a clone, then the fraction of aggregates containing clones should increase, according to "one-hit" Poisson statistics, as $1-e^{-n}$, where $n$ is the average number of stem cells added. Figure 3 shows the results of six experiments in which increasing numbers of live cells were added to nitrogen mustard aggregates. The experimental points are con-
TABLE 2

Cloning Efficiency of Dissoctated Hydra Cells and Stem-Cell Line ${ }^{a}$

\begin{tabular}{lc}
\hline Source of stem cells & $\begin{array}{c}\text { Clones formed/in- } \\
\text { put }(1 \mathrm{~s}+2 \mathrm{~s})\end{array}$ \\
\hline $\begin{array}{l}\text { Dissociated cells from normal } \\
\text { hydra }\end{array}$ & 0.052 \\
Average of 14 determinations & $0.044^{b}$ \\
$\quad$ Data from Fig. 3 & \\
Stem cell line & 0.063 \\
Fifth transfer generation & 0.067 \\
Sixth transfer generation & \\
\hline
\end{tabular}

${ }^{a}$ Normal hydra or NM aggregates carrying the stem cell (David and Murphy, in preparation) were dissociated, and aliquots were assayed for cloneforming cells. Parallel samples were maceraled, and the number of interstitial cells $(1 s+2 s)$ was counted. Results for normal tissue were determined using the $P(O)$ procedure; results for stem-cell line by using the total clone procedure. See Materials and Methods.

${ }^{b}$ Results in Fig. 3 yielded a value of 1 cloneforming cell/165 total hydra cells in the body column; $14 \%$ of these cells are $1 \mathrm{~s}+2 \mathrm{~s}$ (David and Gierer, 1974). Thus, there is 1 clone-forming cell/23 $(1 \mathrm{~s}+2 \mathrm{~s})$ or 0.044 .

sistent with one-hit statistics, assuming that one cell per 165 total cells in the original live cell suspension can found a clone. The results do not match a calculated curve based on the assumption that two cells are required to found a clone. Assumptions that more than two cells are required for each clone match the data even less well.

The results in Fig. 3 also show that the cloning efficiency of stem cells is the same in 1- and 6-day NM host tissues. Since host tissue 6 days after NM treatment contains less than $5 \%$ of the interstitial cells present in a 1-day NM-treated host, this result suggests that the presence of interstitial cells in host tissue does not markedly affect the ability of added stem cells to found clones. The number of clones obtained from a live-cell suspension is, however, influenced by cell losses during reaggregation. Normally, about $50 \%$ of the input cells are recovered in aggregates (Gierer $e t$ $a l ., 1972$ ), and these conditions yield one clone per 165 input cells. In a few experiments in which cell losses were noticeably 


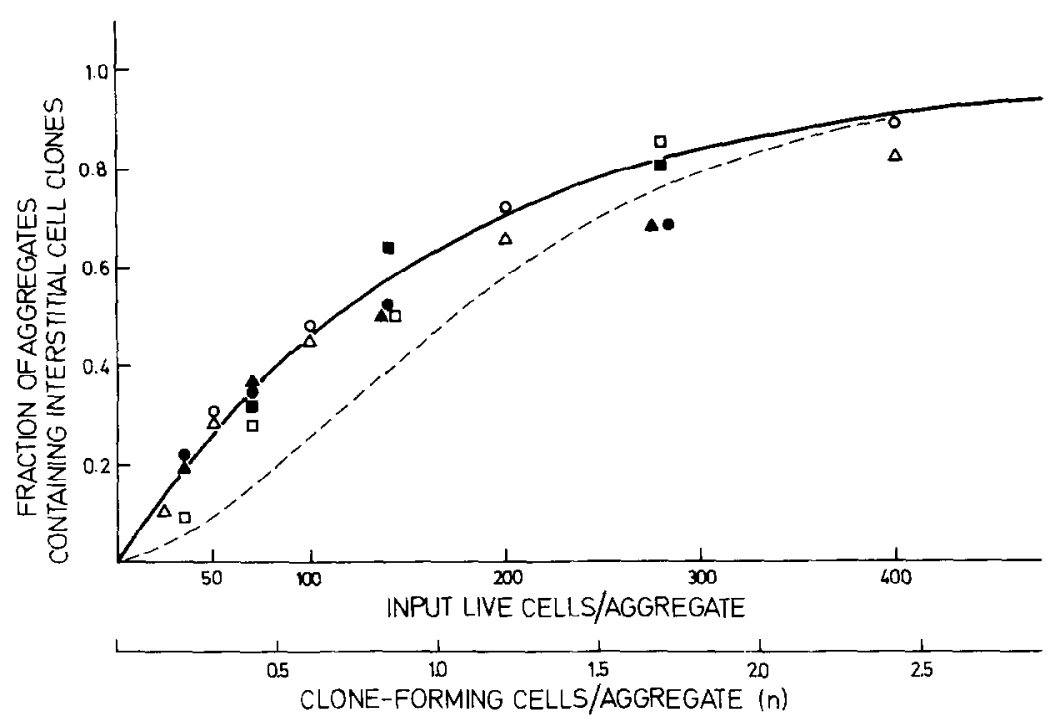

FIG. 3. Formation of interstitial cell clones by live-cell suspensions. Twenty to thirty aggregates were prepared at each input live-cell concentration and were stained for interstitial cell clones after 8-12 days of growth. The results of six independent experiments are presented in the figure. Darkened symbols $(\boldsymbol{\Lambda}, \boldsymbol{\bullet}, \boldsymbol{\nabla})$ indicate experiments using 1-day NM host tissue; blank symbols $(\triangle, O, \square)$ indicate experiments using 7-day pretreated NM host tissue. Control aggregates in each experiment containing no input live cells contained no interstitial cell clones. These values have been deleted from the figure for the sake of clarity. The solid line indicates the fraction of aggregates containing clones based on "one-hit" Poisson statistics: $1-P(0)=$ $1-e^{-n}$, where $P(0)$ is the fraction of aggregates containing no clones, $1-P(0)$ is the fraction containing clones, and $n$ is the average number of clone-forming cells per aggregate. The dotted line indicates the fraction of aggregates containing clones, assuming that two cells are required to found a clone.

higher, the number of input cells per clone was correspondingly higher. Use of a simple isotonic salt solution (Haynes and Burnett, 1963) for preparation of cells leads to systematically greater cell losses during reaggregation and to estimates of 400-600 input cells per clone.

\section{Nerve and Nematocyte Differentiation in Stem Cell Clones}

To determine if individual stem cells can give rise to both nerves and nematocytes, we have examined the types of differentiated cells in clones. Clone-derived cells were distinguished from host cells by labeling aggregates with $\left[{ }^{3} \mathrm{H}\right]$ thymidine. To be certain that any labeled clone cells found in an aggregate were derived from a single stem cell, this experiment was performed under conditions in which less than $20 \%$ of the aggregates contained even one clone, and, thus, fewer than $3 \%$ could have contained two clones.
The results of two independent experiments are presented in Table 3 . In one, four clones were found in 23 aggregates; in the second, five clones were found in 33 aggregates. Each of the nine clones contained stem cells, differentiating nematoblasts, and differentiated nerve cells, thus demonstrating that single stem cells have the potential to differentiate both cell types. Control aggregates to which no live cells were added contained no interstitial cells, nematoblasts, or labeled nerve cells. Furthermore, 42 of the aggregates to which live cells were added, but which had received no stem cell, contained no interstitial cells, no nematoblasts, and no labeled nerve cells. These aggregates serve as further controls. Four aggregates (8b, $3 \mathrm{~b}, 4 \mathrm{a}, 4 \mathrm{~b})$ yielded anomalous results. They contained small numbers of labeled nerve cells and either no interstitial cells or very few interstitial cells. The result cannot be easily dismissed as faulty identi- 
TABLE 3

Nerve and Nematocyte Difrerentiation in Stem-Cell Clones ${ }^{a}$

\begin{tabular}{|c|c|c|c|c|c|c|c|c|c|}
\hline & \multirow{4}{*}{$\begin{array}{l}\text { Experiment } \\
-\mathrm{I}^{-}\end{array}$} & \multirow{4}{*}{$\begin{array}{c}\text { Clone } \\
\text { No. } \\
\\
\\
- \\
1 \mathrm{a}\end{array}$} & \multicolumn{5}{|c|}{ Interstitial cell clusters } & \multirow{3}{*}{$\begin{array}{c}\text { Nemato- } \\
\text { blasts } \\
\text { and } \\
\text { nemato- } \\
\text { cytes }\end{array}$} & \multirow{4}{*}{$\begin{array}{c}\text { Nerve } \\
\text { cells } \\
20\end{array}$} \\
\hline & & & \multicolumn{2}{|c|}{ Stem cells } & \multicolumn{3}{|c|}{$\begin{array}{l}\text { Nematocyte } \\
\text { precursors }\end{array}$} & & \\
\hline & & & 1 & 2 & 4 & 8 & 16 & & \\
\hline \multirow{9}{*}{$\begin{array}{l}\text { Aggregates containing } \\
\text { clones }\end{array}$} & & & 12 & 24 & 14 & 11 & 2 & 2 & \\
\hline & & $3 \mathbf{a}$ & 8 & 28 & 5 & 3 & 5 & 45 & 21 \\
\hline & & $8 a$ & 9 & 8 & 6 & 2 & 1 & 3 & 17 \\
\hline & & $9 \mathrm{~b}$ & 4 & 36 & 10 & 1 & 2 & 38 & 39 \\
\hline & II & $7 a$ & 20 & 18 & 6 & 0 & 0 & 3 & 57 \\
\hline & & $8 \mathbf{a}$ & 8 & 20 & 4 & 1 & 0 & 6 & 23 \\
\hline & & $10 \mathrm{~b}$ & $12^{b}$ & $16^{b}$ & $4^{b}$ & $4^{b}$ & 0 & 0 & 31 \\
\hline & & $15 b$ & 9 & 8 & 3 & 2 & 0 & 4 & 25 \\
\hline & & $16 \mathrm{~b}$ & 20 & 44 & 4 & 1 & 1 & 19 & 50 \\
\hline \multirow{4}{*}{$\begin{array}{l}\text { Aggregates containing } \\
\text { rare clone cells }\end{array}$} & I & $8 b$ & 0 & 0 & 0 & 0 & 0 & 0 & 8 \\
\hline & II & $3 b$ & 0 & 1 & 1 & 1 & 0 & 0 & 12 \\
\hline & & $4 a$ & 0 & 0 & 0 & 0 & 0 & 0 & 7 \\
\hline & & $4 \mathrm{~b}$ & 0 & 1 & $2^{b}$ & $1^{b}$ & 1 & 16 & 3 \\
\hline \multirow{3}{*}{$\begin{array}{l}\text { Aggregates } \\
\text { no clones }\end{array}$} & I & & & & & & & & \\
\hline & $\begin{array}{c}\text { (17 aggregates) } \\
\text { II }\end{array}$ & & 0 & 0 & 0 & 0 & 0 & 0 & 0 \\
\hline & (25 aggregates) & & 0 & 0 & 0 & 0 & 0 & 0 & 0 \\
\hline
\end{tabular}

"Clones were prepared in pretreated NM host aggregates so that only $15-20 \%$ of the aggregates contained clones. Aggregates were labeled with [ $\left.{ }^{3} \mathrm{H}\right]$ thymidine $(0.01 \mu \mathrm{Ci}$ /aggregate) on Days 10,11 , and 12 of clone growth, and macerations were made on Day 14 of each aggregate individually. Following autoradiography, macerations were completely scanned for labeled interstitial cells, nematoblasts, and nerve cells. The number of interstitial cell clusters of each size class, the total number of nematoblasts, and the total number of nerve cells are recorded in the table. The results of two experiments are presented in which 9 aggregates contained clones, 4 aggregates contained a few labeled cells not identifiable as clones, and 42 aggregates contained no clones. Control aggregates to which no live cells were added, but which otherwise were identically prepared and scored, were found to contain no labeled interstitial cells, nematoblasts, or nerve cells.

${ }^{\circ}$ Results in which some cells were unlabeled.

fications, in regard to either cell type or degree of labeling, since the accuracy and the resolution of the procedure is rather high. Possibly, such cells are derived from clones with a limited capacity for proliferation or differentiation. However, a more likely explanation appears to be the loss of a major portion of the clone as a result of fragmentation of the aggregate into separate hydra at late stages of development.

It could be argued that the multipotent clones observed in Table 3 arose from clumps of input cells containing a determined nerve and a determined nematocyte stem cell. Standard preparations of dissociated cells contain up to $5 \%$ of interstitial cells as pairs and about $0.5 \%$ as clumps of $3-5$ cells. Such clumps appear microscopically to arise by incomplete dissociation of interstitial cell clusters which occur in vivo. Since cells in such clusters are siblings derived from a single parent cell (David and Gierer, 1974; Slautterback and Fawcett, 1959), they would not affect the multipotency conclusion. However, if such cell clumps arise by reassociation of uniquely determined stem cells during cell preparation in vitro, they could give rise to apparently multipotent clones. Since only multipotent clones are observed (Table 3), it is also necessary to assume: (1) that all single stem cells are inactivated by the 


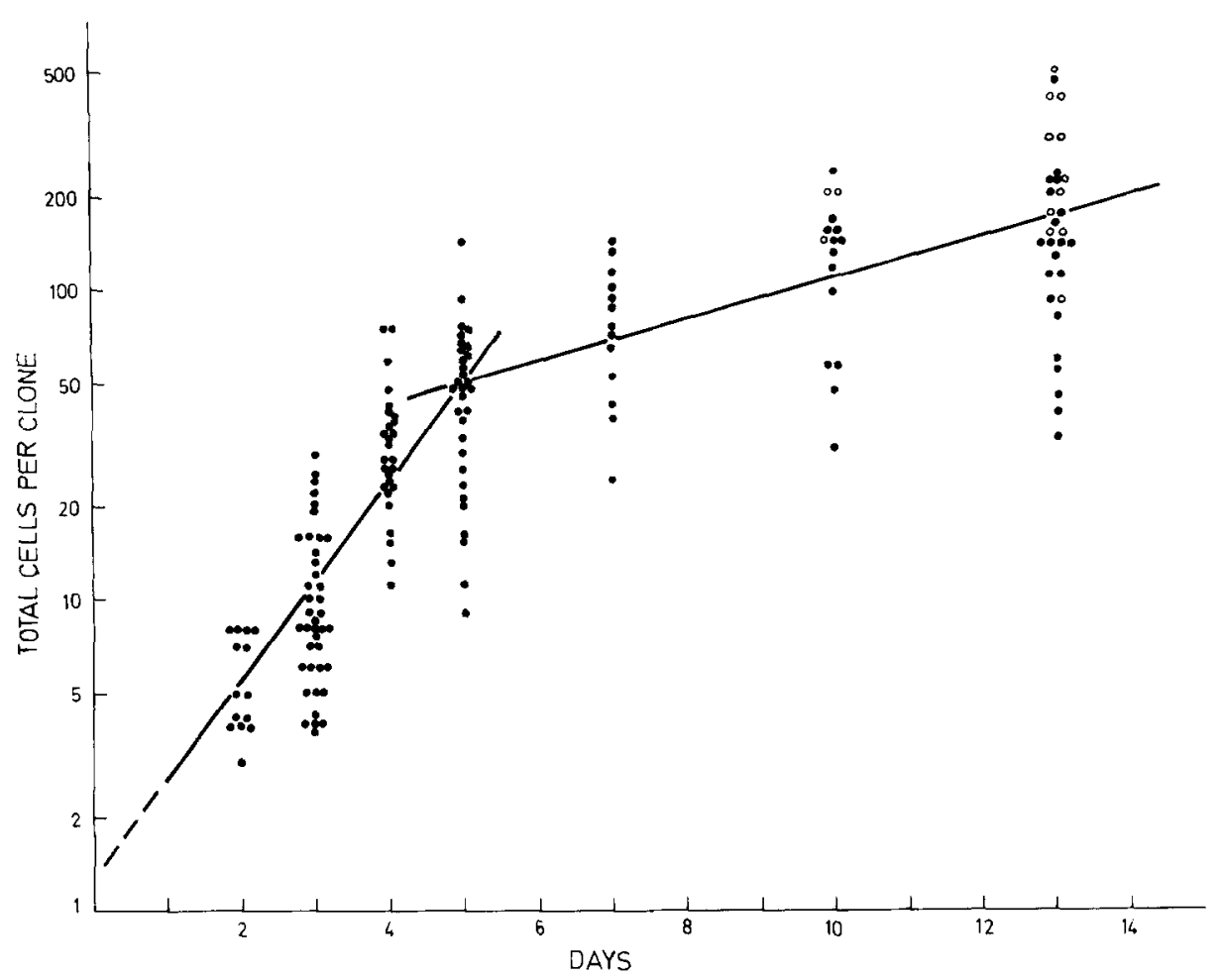

FIG. 4. Growth of interstitial cell clones. Clones were prepared in 6-day pretreated NM host aggregates. Starting on Day 2, aggregates were stained with toluidine blue, and the number of interstitial cells and differentiating nematoblasts was counted in individual clones. Clone nerve cells cannot be distinguished from host nerve cells and, therefore, are not included in the total number of cells per clone. Each point represents one clone. Blank circles are estimated counts for large clones in which cells were too tightly packed to be scored exactly.

dissociation procedure; and (2) that each clump contains both nerve and nematocyte stem cells. Both assumptions are implausible. Dissociated single hydra cells are generally viable (Gierer et al., 1972), and, thus, it appears unlikely that single stem cells are selectively inactive. Furthermore, the postulated reassociation of stem cells would have to be essentially complete under the conditions of cell preparation ( 20 min; stem cell concentration of 200-300 cells $/ \mathrm{ml}$ ), and the cloning efficiency would have to be $100 \%$ in order to obtain the number of multipotent clones observed. In view of these difficulties, it appears unlikely that the clones in Table 3 arose from clumps in the input-cell suspension. Moreover, the growth curve of clones (Fig. 4) extrapolates back to 1-2 cells/clone at $t_{0}$, as expected if clones arise from single cells of an unsynchronized population.

The results in Table 3 demonstrate that single hydra stem cells are capable of founding clones containing both differentiated nerves and nematocytes. To increase the number of clones examined and to score for the presence of several nematocyte types in one clone, we examined an additional 50 clones by toluidine blue staining of whole-mounted aggregates in which interstitial cells and clusters of differentiating nematoblasts can be identified. We only scored clones in experiments in which the fraction of aggregates containing clones was less than $35 \%$ to minimize confusion of single clones with two closely spaced clones.

Results were obtained from seven inde- 
pendent experiments stained after 10-13 days of clone growth, at which time the average clone size was $100-200$ total cells. The occurrence of stem cells (single or paired large interstitial cells), determined clusters of interstitial cells (clusters of 4,8 , 16), and clusters of differentiating nematoblasts was scored in each clone. Of 50 such clones examined, 48 contained stem cells, and 49 contained cells at some stage of nematocyte differentiation. Only one clone had the appearance of a putative nerve-cell clone, i.e., stem cells and no differentiating nematocytes. Together with the results in Table 3 , showing differentiating nerves and nematocytes in all nine clones examined, these results constitute strong proof that the interstitial stemcell population in hydra is homogeneous, or very nearly homogeneous, with regard to its potential to differentiate both nerves and nematocytes. Furthermore, 10 clones in this experiment contained more than one type of differentiating nematoblast, demonstrating that stem cells are probably multipotent in regard to nematocyte differentiation as well.

\section{Growth of Clones and the Initiation of Dif- ferentiation}

The cell composition of interstitial cell clones was examined in aggregates stained with toluidine blue at various times after cloning (Fig. 2). On Day 2, clones consist of only large interstitial cells, single or paired. By Day 4, nests of four and eight interstitial cells begin to appear as the first stages in nematocyte differentiation. By 10-12 days, clones contain 60-250 cells of various types, including clusters of interstitial cells at various stages of nematocyte differentiation as well as large interstitial cells. There are also 20-50 nerve cells in clones at this stage, as disclosed by labeling with $\left[{ }^{3} \mathrm{H}\right]$ thymidine to distinguish clone nerve cells from host nerve cells (Table 3 ).

Growth of stem-cell clones occurs by proliferation of stem cells within the clone; differentiating daughter cells do not contribute to further clone growth. In the particular case of interstitial-cell clones, daughter cells initiating nematocyte differentiation continue to contribute to increases in cell number for several days after determination, since the first stage of nematocyte differentiation includes 2-4 cell divisions (about 3 days). Thus, we anticipate a change in the growth rate of clones about 3 days after nematocyte determination when clusters of nematocyte precursors have completed cell division and initiated differentiation. We have used this fact to define the onset of nematocyte determination in clones.

Figure 4 shows quantitatively the total number of cells per clone (excluding nerve cells) for a large number of clones examined at various times after the onset of clone growth. For the first 5 days, the total number of cells per clone doubles about once per day, in agreement with the average cell cycle time of 18-24 hours for interstitial cells in normal animals (Campbell and David, 1974). Beginning on the fifth day, there is a sharp decrease in the growth of cell numbers, which coincides with the appearance of differentiating nematoblasts in clones. Subtracting 3 days from the observed break in the curve in Fig. 3 indicates that some daughter cells were determined to differentiate nematocytes as early as Day 2 of clone growth, at a time when clones contained only 4-8 cells.

\section{DISCUSSION}

\section{An Assay for Stem Cells in Hydra}

David and Gierer (1974) demonstrated the existence of interstitial stem cells in hydra having the capacity to produce precursors for nerve and nematocyte differentiation as well as the capacity to reproduce themselves. In the present study we have developed a direct clonal assay for interstitial stem cells. The procedure is essentially a feeder-layer technique involving growth of added live cells in host tissue 
which has been inactivated by nitrogen mustard treatment. Clones of interstitial cells and differentiating nematoblasts and nerve cells are formed in such hosts when limiting numbers of live cells are added. The clones arise from single interstitial cells as shown by the facts that the incidence of clones follows one-hit Poisson statistics (Fig. 3) and that a pure interstitial cell line (David and Murphy, in preparation) yields clones with the same efficiency as mixed populations of hydra cells. The reproducibility of the cloning procedure is high, and it can be used to assay stem cells quantitatively, by counting the clones directly or the fraction of aggregates containing clones.

The use of NM aggregates as hosts for cloning other dividing cell types in hydra appears feasible if suitable procedures can be found to distinguish the cloned epithelial and gland cells from similar cells in the host aggregate. In addition, the NM aggregates can be used to assay interstitial-cell determination in vivo since determined cells will continue differentiation in such hosts (David, unpublished). It may also be possible to use NM aggregates as the intermediate host in attempts to adapt stem cells to growth in tissue culture conditions (Sato and Yasumura, 1966).

\section{Estimate of Stem Cells per Hydra}

Based on the requirements for interstitial-cell differentiation in hydra, David and Gierer (1974) estimated a total of 3600 interstitial stem cells per hydra or about $4 \%$ of the total of 90,000 cells in the body column of standard animals. The fraction of total cells initiating clones is $1 / 165$ or $0.6 \%$ (Fig. 3). This is a minimum estimate which includes cell losses due to the dissociation and aggregation procedures as well as the inability of some stem cells to initiate clones. Corrections can be made for some of these losses. Gierer et al. (1972) have shown that only about $50 \%$ of cells in a suspension are incorporated into tissue structures during aggregation. The re- mainder are sloughed off in the first minutes of aggregate preparation or are in the core of the aggregate and fail to get incorporated into the two-layered epithelium which forms during the first hours of aggregation. Correcting for this loss, the cloning experiments suggest that $1.2 \%$ of hydra cells are stem cells.

The difference between 1.2 and $4 \%$ might be ascribed to the poor cloning efficiency of stem cells under our conditions due to nonideal dissociation procedures, medium, etc. This explanation assumes that all viable stem cells entering aggregates can form clones. An alternative explanation is also possible. Some viable stem cells may initiate differentiation to the exclusion of stem-cell proliferation, thus extinguishing a potential clone. Vogel et al. (1969) have calculated that, for conditions such as ours, about two-thirds of the input stem cells will fail to form clones which would raise the value of 1.2 to $3.6 \%$, in good agreement with the estimate of $4 \%$ derived from in vivo measurements.

In vivo, stem cells constitute about $40 \%$ of the population of large interstitial cells occurring as single cells and in pairs (David and Gierer, 1974). Correcting this value for cell loss $(\times 1 / 2)$ and cloning efficiency and/ or clone extinction $(\times 1 / 3)$ leads to an estimate that $7 \%$ of the input cells of the classes $1 \mathrm{~s}$ and $2 \mathrm{~s}$ can form clones. This value agrces reasonably well with measured values of 4.4-6.7\% (Table 2).

\section{Multipotency of Stem Cells}

The results in Table 3 demonstrate the occurrence of both nerve and nematocyte differentiation in nine clones derived from single stem cells. No clones were found in which only nerve or nematocyte differentiation occurred. These results, together with the much larger sample of clones each containing differentiating nematocytes, demonstrate that interstitial stem cells are multipotent and can differentiate into both nerves and nematocytes. The present results provide no evidence for 
subpopulations of determined stem cells as postulated in the hemopoietic system (Metcalf and Moore, 1971), the differentiation potential of which is limited to one cell type, but which still retain sufficient proliferative capacity to form clones.

The role of "position" in determining the differentiation of stem-cell clones in aggregates is unclear in the present experiments. In normal animals, nerve differentiation occurs primarily near the hypostome and basal disk, whereas nematocyte differentiation occurs in the gastric region (David and Gierer, 1974). In principal, such regionalization might have caused predominantly nerve or nematocyte differentiation in clones developing in the corresponding regions of aggregates and, thus, might have obscured the observation of multipotent clones. In particular, since most clones appear as compact groups of cells in the "gastric region" of aggregates, we might have observed predominantly nematocyte rather than nerve differentiation in our clones. The reverse result was, in fact, obtained, particularly in the second experiment (Table 3). At present, we do not understand the meaning of this observation. It may be that the observed regionalization of differentiation in normal animals does not reflect regionalization at the level of determination. Or, it may be that other environmental effects such as starvation, which enhances nerve differentiation, play a predominant role in aggregates.

The possibility that interstitial cells constitute an "embryonic reserve" capable of differentiating into all cell types in adult hydra has been suggested many times (Tardent, 1963; Lentz, 1966; Burnett, 1966). The present experiments do not resolve this issue. However, the availability of a cloned population of stem cells containing no viable epithelial and gland cells (Table 2) offers an experimental approach to testing rigorously the postulated totipotency of interstitial cells. Similarly, investigation of the fate of cloned stem cells transplanted into sexual hydra can be used to determine whether multipotent stem cells are identical to interstitial cells differentiating egg and sperm in sexual hydra (Brien, 1961).

\section{Control of Stem-Cell Proliferation and Differentiation in Clones}

We have identified a class of multipotent stem cells in hydra. Each of these cells must decide, once in each cell cycle, to proliferate further stem cells (self-renewal) or to differentiate. The frequency of these events in any stem cell generation can be characterized by a self-renewal probability $(p)$ and a differentiation probability $(1-p)$. In the following, we wish to summarize the existing evidence for feedback control of stem-cell proliferation in hydra and to suggest how these controls may regulate the self-renewal probability of stem cells during the growth of clones.

The number of stem cells in normal hydra is remarkably constant for a variety of different growth conditions (Bode et al., 1976). This suggests the existence of a homeostatic mechanism regulating the concentration of stem cells in hydra tissue. Recently, Bode et al. (1976) obtained direct evidence for stem-cell homeostasis by showing that experimentally-induced depletion of stem cells in hydra tissue causes an increased growth rate in the stem-cell population and eventual recovery of the normal stem-cell concentration in tissue. In addition, they were able to show that the homeostatic mechanism operated by altering the self-renewal probability of stem cells: Depletion of stem cells increased the self-renewal probability; saturation decreased it. Applying this result to the growth of stem cell clones, we would anticipate $p$ values approaching 1.0 in early (small) clones and $p$ values approaching 0.6 in late (large) clones in which conditions approach those in normal tissue (David and Gierer, 1974). Concurrently, we anticipate little or no differentiation in early clones and more extensive 
differentiation in late clones. The time during clone growth at which the change in $p$ occurs depends on the range of the homeostatic feedback signal. If a longrange signal (such as a highly diffusible molecule) is involved, the strength of the signal perceived by a stem cell will reflect the stem-cell concentration averaged over a large area and the shift in $p$ will not occur until clones are quite large. In the case of a short-range signal, the strength of the feedback perceived by a stem cell will reflect the local concentration of stem cells, and the change in $p$ will occur when clones are small.

A striking feature of interstitial cell clones is the occurrence of extensive nematocyte determination after 2 days of clone growth when clones consist of only 4-8 cells (Fig. 4). This result excludes the possibility that the feedback signal is long range and suggests that short-range feedback between neighboring stem cells may he regulating the value of $p$ in clones. In agreement with this suggestion, we have recently shown that the self-renewal probability decreases from $\geq 0.8$ in 0 -3-day clones to 0.6 in 14-day clones (David and MacWilliams, in preparation).

The authors thank Dr. H. K. MacWilliams for helpful discussions and critical reading of the manuscript. The research was supported by NIH Grant No. GM 11301 and a Career Development Award (No. FRA-132) from the American Cancer Society.

\section{REFERENCES}

Brien, P. (1961). Elude d'Hydra pirardi. Origine et repartition des nematocystes. Gametogenese. Involution postgametique. Evolution reversible des cellules interstitielles. Bull. Biol. Fr. Belg. 95, 301-364.

Bode, H. G., Flick, K. M., and Smith, G. S. (1976). Regulation of interstitial cell differentiation in Hydra attenuata. I. Homeostatic control of interstitial cell population size. J. Cell Sci. 20, 29-46.

Burnett, A. L. (1966). A model of growth and cell differentiation in hydra. Amer. Natur. 100, 165189

Campbell, R. D., and David, C. N. (1974). Cell cycle kinetics and development of Hydra attenuata. II.
Interstitial cells. J. Cell Sci. 16, 349-358.

DAvid, C. N. (1973). A quantitative method for maceration of Hydra tissue. Wilhelm Roux Arch. Entwicklungsmech. Organismen 171, 259-268.

David, C. N., and Campbell, R. (1972). Cell cycle kinetics and development of Hydra attenuata. I. Epithelial cells. J. Cell Sci. 11, 557-568.

David, C. N., and Challoner, D. (1974). Distribution of interstitial cells and differentiating nematocytes in nests in Hydra attenuata. Amer. Zool. 14, 537.542 .

David, C. N., and Gierer, A. (1974). Cell cycle kinetics and development of Hydra attenuata. III. Nerve and nematocyte differentiation. J. Cell Sci. 16, 359-375.

DAvis, L. E. (1969). Differentiation of neurosensory cells in hydra. J. Cell Sci. 5, 699-726.

DAvis, L. E. (1971). Differentiation of ganglionic cells in hydra. J. Exp. Zool. 176, 107-128.

Diehl, F., and Burnett, A. L. (1964). The role of interstitial cells in the maintenance of hydra. I. Specific destruction of interstitial cells in normal, sexual and non-budding animals. J. Exp. Zool. 155, 253-259.

Gierer, A., Berking, A., Bode, H., David, C. N., Flick, K., Hansmann, G., Schaller, C., and TRENkNER, E. (1972). Regeneration of hydra from reaggregated cells. Nature New Biol. 239, 98-101.

Haynes, J., and Burnett, A. L. (1963). Dedifferentiation and redifferentiation of cells in Hydra viridis. Science 142, 1481-1483.

Lentz, T. L. (1966). "The Cell Biology of Hydra." North-Holland, Amsterdam.

Metcalf, D., and Moore, M. A. S. (1971). "Haemopoietic Cells." North-Holland, Amsterdam.

Sato, G. H., and Yasumura, Y. (1966). Retention of differentiated function in dispersed cell culture. Trans. N. Y. Acad. Sci. 28, 1063-1079.

Slautterback, D. B., and Fawcett, D. W. (1959). The development of the cnidoblasts of hydra. An electron microscope study of cell differentiation. J. Biophys. Biochem. Cytol. 5, 441-452.

Till, J. E., andMcCulloch, E. A. (1961). A direct measurement of the radiation sensitivity of normal mouse bone marrow cells. Radiat. Res. 14 , 213-222.

TARDENT, P. (1963). Regeneration in the hydrozoa. Biol. Rev. 38, 293-333.

Trenkner, E., Flick, K., Hansmann, G., Bode, H., and Bode, P. (1973). Studies on hydra cells in vitro. J. Exp. Zool. 185, 317-326.

Vogel, H., Niewisch, H., and Matioli, G. (1969). Stochastic development of stem cells. $J$. Theor. Biol. 22, 249-270.

Wolpert, L. (1971). Positional information and pattern formation. Curr. Top. Develop. Biol. 6, 183224 . 